Bundesgesundheitsbl 2015 · 58:1171-1174

DOI 10.1007/s00103-015-2215-5

Online publiziert: 15. August 2015

๑) Springer-Verlag Berlin Heidelberg 2015

CrossMark

Geschäftsstelle der Kommission Umweltmedizin

\title{
Empfehlung der Kommission Umweltmedizin am Robert Koch-Institut zu Moderne Lichtquellen
}

organischen Leuchtdioden (OLED: organic light emitting diode) zu erwarten.

Dies hat zu Fragen nach der ,gesundheitlichen Unbedenklichkeit moderner Lichtquellen " geführt. Zu betrachten sind hier hauptsächlich das Lichtspektrum der Lichtquelle, die Tages- bzw. Nachtzeit der Lichtexposition und ihre Dauer sowie der individuelle Chronotyp [10-12, 15].

Über die umweltmedizinische Ebene hinaus sollen ökologische, ästhetische und astrophysikalische Aspekte der Lichtimmission, insbesondere in Ballungsgebieten, nicht unerwähnt bleiben. Das Ausmaß der bestehenden „Lichtverschmutzung" wird bezüglich ihres möglichen Einflusses auf Insekten, Zugvögel, Flora und den „Verlust der Nacht“ zunehmend kritisch gesehen.

Die Kommission hat sich insbesondere mit drei Aspekten moderner Lichtquellen befasst: Melatoninsuppression, Farbwiedergabe und Lichteinwirkung auf das Auge. Dabei geht die Kommission von ty- pischen Beleuchtungs- und Nutzungsszenarien im häuslichen Wohnumfeld aus.

In der im Internet verfügbaren Langfassung der Mitteilung werden Unterschiede der verschiedenen Beleuchtungsarten aufgezeigt und die gesundheitlichen Auswirkungen des Blaulichtanteils detailliert auch unter chronobiologischen Aspekten betrachtet. Auch die Frage von Flimmereffekten wird diskutiert.

Tageslicht, Glühlampen und Halogenglühlampen sind die einzigen Lichtquellen mit kontinuierlichem (vollem) Lichtspektrum, während alle anderen Lichtquellen Licht mit mehr oder weniger ausgeprägten schmal- bis breitbandigen Peaks im blauen und grünen Spektralbereich aussenden.

\section{Spektren unterschiedlicher Lichtquellen}

- Abb. 1
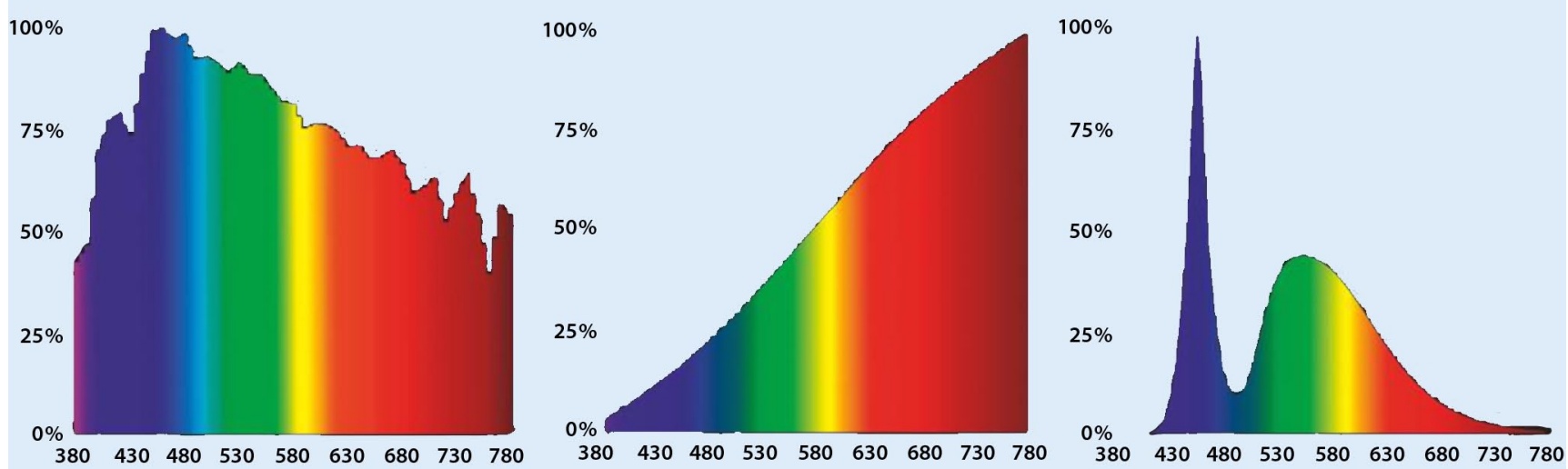

Abb. 1 A Lampenspektren (v.I.n.r.: Tageslicht, Halogenlampe, LED kaltweiss). Abszisse: Wellenlänge (nm), Ordinate: normierte Strahlungsleistung. Mit freundlicher Genehmigung der Fördergemeinschaft Gutes Licht (Licht.de) 


\section{Blaulichtanteil und \\ Melatoninsuppression}

Der menschliche Körper ist - wie die allermeisten anderen Lebewesen auch - auf einen 24-h-Rhythmus eingestellt, der auch als „zirkadianer Rhythmus“ oder „innere Uhr" bezeichnet wird. Dieser Rhythmus regelt den Schlaf-Wach-Zyklus, die Hormonausschüttung, den Zellstoffwechsel, Zellwachstum, Zellteilung und Energiebilanz beim Menschen.

Externe Faktoren, von denen Licht wahrscheinlich der wichtigste ist, können den zirkadianen Rhythmus an den aktuellen Tagesverlauf anpassen [10, 11]. Die Information, wieviel Licht auf das Auge einfällt, wird über ,intrinsisch photosensitive retinale Ganglienzellen“ (ipRGC) an den „master circadian pacemaker" im suprachiasmatischen Nucleus übertragen, dieser steuert die Melatoninsynthese in den Pinealozyten der Zirbeldrüse (Epiphyse). Licht ist - abhängig von Zeitpunkt seiner Einwirkung, Dauer, Intensität und seinen Spektraleigenschaften - in der Lage, die Melatoninsynthese zu hemmen. Blaues Licht im Wellenlängenbereich zwischen 460 und $484 \mathrm{~nm}$ wirkt hier am effizientesten.

Im Fachbericht DIN 67600 „Biologisch wirksame Beleuchtung - Planungsempfehlungen“ " 6] werden eine Beleuchtungsstärke von mindestens 250 Lux (gemessen am Auge des Betrachters) und eine Farbtemperatur von $8000 \mathrm{~K}$ als Mindestvoraussetzungen für biologisch wirksames Licht genannt.

Melatonin hat vielfältige Aufgaben im Körper: Neben seiner Funktion als „Zeitgeber" dient es auch als Antioxidans und als Radikalfänger. Ferner verstärkt es Immunantworten und beeinflusst bestimmte apoptotische Prozesse ([11], S. 54).

Tagsüber dient die Melatoninsuppression zur Steigerung der Aufmerksamkeit und ist gleichzeitig zur Verbesserung des nächtlichen Schlafverhaltens wichtig und sinnvoll, während eine nächtliche Melatoninsuppression - je nach Chronotyp im Allgemeinen nachteilig ist.

Eine längerdauernde abendliche oder nächtliche Lichtexposition mit einem hohen Blaulichtanteil kann daher zu einer verlängerten Melatoninsuppression bis in die Nacht hinein führen.
Nach gegenwärtigem Kenntnisstand spielt eine Melatoninsuppression wahrscheinlich eine wichtige Rolle bei der Entwicklung chronischer Krankheiten. Sie wird mit Schlafstörungen, gastrointestinalen und kardiovaskulären Beschwerden, sowie mit der Inzidenz von Depressionen in Verbindung gebracht 11]. Ferner werden auch Krebserkrankungen der Brust $[4,8,9]$ und der Prostata [13] im Zusammenhang mit einer Melatoninsuppression diskutiert.

Eine dem natürlichen Tagesgang entsprechende Lichtführung mit allmählich ansteigender morgendlicher Lichtexposition, die tagsüber helles tageslichtweißes Licht beinhaltet und zum Abend hin zu gedämpftem, eher rötlichem Licht wechselt, wird von den meisten Personen als angenehm empfunden.

Nach Meinung der Kommission können folgende Empfehlungen gegeben werden:

1. Tagsüber ist chronobiologisch wirksames Licht, das dem Spektrum des Tageslichts entspricht, in der Regel zur Stabilisierung der zirkadianen Rhythmik sinnvoll. Es kann darüber hinaus, insbesondere bei älteren Personen, auch den abendlichen Schlafdruck und das nächtliche Schlafverhalten fördern.

In den Abend- und Nachtstunden ist in der Regel die durch Licht mit hohem Blaulichtanteil bedingte Melatoninsuppression unerwünscht. Hier sollten Lichtquellen mit niedrigem Blaulichtanteil, gewöhnlich mit niedriger Farbtemperatur $(<3300 \mathrm{~K})$, verwendet werden.

2. Der Einfluss einer längeren abendlichen Arbeit an LED-basierten Computermonitoren, Tablets und Smartphones auf die Melatoninproduktion kann aufgrund der noch unzureichenden Datenlage nicht abschließend beurteilt werden.

Aus der wissenschaftlichen Literatur $[5,14]$ liegen Hinweise auf eine entsprechende Melatoninsuppression vor. In einigen Geräten besteht allerdings die Möglichkeit, mittels einer speziellen „App“ oder über die Systemkonfiguration die Farbtemperatur des Monitors einzustellen und damit den Blauanteil des Lichts zu verringern.
Das abendliche Fernsehen mittels LED-basierter Fernsehgeräte kann gleichfalls nicht abschließend beurteilt werden. Eine einzelne Studie [7] aus dem Jahr 2013 fand keinen Effekt auf die Melatoninsynthese bzw. -inhibition und erklärt dies mit dem üblicherweise größeren Abstand zwischen Bildschirm und Betrachter.

Nach Auffassung der Kommission sollten zukünftig auch Spielkonsolen, Spielzeuge, beleuchtete Anzeigetafeln, Nachtbeleuchtungen etc. in die Überlegungen zur (unerwünschten nächtlichen) Melatoninsuppression einbezogen werden.

\section{Farbwiedergabe}

Überwiegend stammt die Lichtenergie, die wir aufnehmen, aus Objekten, deren Lichtenergie sich als Produkt aus Reflektions- und Absorptions- bzw. Transmissionseigenschaften und dem Energiespektrum der beleuchtenden Lichtquelle(n) darstellt. Der Farbreiz, als der ein Farbstoff dem Betrachter erscheint, hängt daher wesentlich von der Spektralverteilung der Beleuchtung, d. h. von der Art der Lichtquelle ab. Für die Farbwiedergabe sind kontinuierliche Spektren, wie sie das Licht der „alten“ Glühlampe hatte, von Vorteil. Licht dieser Quelle hat den allgemeinen Farbwiedergabeindex $\mathrm{Ra}=100$. Auch wenn mittlerweile viele LED Lampen einen Farbwiedergabeindex von 90 und höher erreichen, unterscheidet sich das Spektrum deutlich von dem einer Glühfadenlampe. Der Farbwiedergabe-index ist ein stark vereinfachendes Distanzmaß, um die spektrale Verteilung (Farbwiedergabe) von acht unter Testbedingungen beleuchteten DIN-Normtafeln in Relation zu einer Normlichtquelle zu beschreiben.

\section{Empfehlungen:}

1. Visuelle Experimente haben gezeigt, dass der Farbwiedergabeindex die subjektiv wahrgenommenen Farbwiedergabe bei unterschiedlichen Leuchtmitteln nicht immer einwandfrei beschreibt, d. h. von der subjektiv wahrgenommenen Farbwiedergabe abweicht. Die mit neuen Leuchtmitteln verbundene Veränderung der 
spektralen Verteilung der Lichtemission sollte wegen der hohen Prävalenz von Farbfehlsichtigkeit, aus genetischen Gründen insbesondere für Männer relevant, in Bezug auf mögliche Schwierigkeiten bei der Farbunterscheidung geprüft werden.

2. Da weiterhin die subjektive Farbempfindung von Raumlicht zugleich von der Beleuchtungsstärke und der Farbtemperatur abhängt (Kruithof-Beziehung), müssen die Verbraucher differenziert über die spektrale Lichtverteilung der neuen Leuchtmittel informiert werden, um eine den Bedürfnissen angepasste Auswahl treffen zu können.

\section{Lichteinwirkungen auf das Auge}

Potentielle gesundheitliche Risiken durch Lichtquellen aller Art bestehen dann, wenn die Augen einfallendes energiereiches Licht aufnehmen, welches durch die Augenlinsen weiter fokussiert wird. Die resultierenden zellulären Wirkungen einer Lichtexposition hängen von der Wellenlänge, der Beleuchtungsstärke, vom exponierten Zelltyp und von der spezifischen molekularen Reaktion ebenso $\mathrm{ab}$ wie von der Verfügbarkeit, der Geschwindigkeit und der Wirksamkeit der körpereigenen Schutzreaktionen (z. B. Verschluss der Lider, Blinzeln, Pupillenverengung, Hell-Dunkel-Adaptation, der Einwirkdauer usw.).

Die Lichtstärke moderner LEDs ist so groß, dass bei direktem Hineinschauen aus geringem Abstand (etwa $20 \mathrm{~cm}$ oder weniger) die Netzhaut der Augen vorübergehend oder dauerhaft geschädigt werden kann. Einzelheiten können im BAuA-Abschlussbericht „Photobiologische Sicherheit von Licht emittierenden Dioden (LED)“ " [2] nachgelesen werden. Der Lidschlussreflex funktioniert übrigens zuverlässig nur bei einem Fünftel der Bevölkerung bei monochromatischem Licht. Mögliche Risiken aus einer Verminderung des Lidschlussreflexes bei neurodegenerativen Erkrankungen (insbesondere im höheren Alter) sollten beachtet werden.

Bei speziellen UV-LEDs und IR-LEDs müssen zusätzlich schädigende Expositionswirkungen von Strahlung des kurz- welligen ultravioletten Lichts (UV, Risiko: photochemische Netzhautschädigung) und des langwelligen Infrarotlichts (Wärmestrahlung, Risiko: thermische Netzhautschädigung) beachtet werden, die bei handelsüblichen LEDs keine Rolle spielen.

\section{Empfehlung:}

Ausdrücklich unterstützt die Kommission Umweltmedizin die Forderung der französischen „Agence nationale de sécurité sanitaire de l'alimentation, de l'environnement et du travail“ (ANSES [1,3], Käufer und Nutzer über mögliche photobiologische Risiken (Gefahr der Blendung und Netzhautschädigung beim direkten Hineinsehen in LED-Strahler) zu informieren. Eine leicht verständliche Beschriftung auf der Verpackung entsprechender Produkte sollte auf die zugehörige Risikoklasse (photobiological safety risk group) dieser Produkte hinweisen.

Das gilt insbesondere für LEDs im Haushalt ab einschließlich der Risikoklasse 1, wenn die Gefahr einer direkten Lichtexposition aus weniger als $20 \mathrm{~cm}$ Entfernung gegeben ist.

Für neue Lichttechnologien z. B. LED/ Laser-basierte Scheinwerfer) sollte die gesundheitliche Verträglichkeit vor Markteinführung geprüft werden.

Die Empfehlungen wurden von der Kommission Umweltmedizin am Robert Koch-Institut auf ihrer Sitzung am 17. Juni 2015 verabschiedet.

\section{Federführung}

Dr. Matthias Otto (Gemeinnützige Kinderumwelt GmbH), Dr. Michael Schümann (vormals Behörde für Gesundheit und Verbraucherschutz Freie und Hansestadt Hamburg), Prof. Dr. Caroline Herr (Bayerisches Landesamt für Gesundheit und Lebensmittelsicherheit) in Verbindung mit dem externen Sachverständigen Dr. Bernhard Brenner (Bayerisches Landesamt für Gesundheit und Lebensmittelsicherheit), Dr. Hildegard Niemann (Geschäftsstelle der Kommission Umweltmedizin am Robert Koch-Institut)

\section{Kommissionsmitglieder}

Prof. Dr. K.-Christian Bergmann (Allergie-Centrum, Charité Universitätsmedizin Berlin), Prof. Dr. W. Dott (Universitätsklinikum Aachen, Institut für Hygi- ene und Umweltmedizin), Prof. Dr. Th. Eikmann (Universität Gießen, Institut f. Hygiene u. Umweltmedizin), Dr. J. Heinrich (Helmholtz Zentrum München, Epidemiologie I), Priv.-Doz. Dr. med. Astrid Heutelbeck (Universitätsmedizin Göttingen (UMG), Institut für Arbeits-, Sozialund Umweltmedizin), Prof. Dr. B. Hoffmann (Heinrich-Heine-Universität Düsseldorf, Institut für umweltmedizinische Forschung), Prof. Dr. C. Hornberg (Universität Bielefeld, Fakultät für Gesundheitswissenschaften), Dr. K. Leitner (Ehemalige Beigeordnete Generaldirektorin WHO, Genf), Prof. Dr. V. Mersch-Sundermann (Universität Freiburg, Institut Krankehaushygiene u. Umweltmedizin), Prof. Dr. D. Nowak (LMU München, Klinikum Innenstadt, Institut u. Poliklinik für Arbeits- und Umweltmedizin), Prof. Dr. A. Peters (Helmholtz Zentrum München, Epidemiologie II), Prof. Dr. G. A. Wiesmüller (Gesundheitsamt Köln)

\section{Ständige Gäste}

Dr. U. Winkler, K. Höppner M. Sc. (Bundesministerium für Gesundheit), J. Küllmer (Bundesministerium für Umwelt, Naturschutz, Bau und Reaktorsicherheit), Dr. A. Gies, Dr. W. Straff, Dr. A. Neumann, Dr D. Plaß (Umweltbundesamt), Dr. H. Desel (Bundesinstitut für Risikobewertung, Berlin), Dr. Th. Jung (Bundesamt für Strahlenschutz), Dr. Th. Ziese, Dr. M. Faber, Prof. Dr. K. Stark (Robert KochInstitut), Dr. Frank Benkwitz (Vorsitzender der Länderarbeitsgruppe umweltbezogener Gesundheitsschutz, LAUG)

\section{Kontakt über die Geschäftsstelle am RKI}

Robert Koch-Institut

Geschäftsstelle der Kommission Umweltmedizin

Fachgebiet 24: Gesundheitsberichterstattung

Postfach 650261

13302 Berlin, Deutschland

E-Mail:Umweltmedizinkommission@rki. de 


\section{Literatur}

1. ANSES (2010) Effets sanitaires des systèmes d'éclairage utilisant des diodes électroluminescentes (LED). Avis de l'Anses, Rapport d'expertise collective, Saisine $n^{\circ}{ }^{2} 2008-S A-0408^{\prime \prime}$. Agence nationale de sécurité sanitaire de l'alimentation, de l'environnement et du travail. Maisons-Alfort, le 19 octobre 2010. https://www.anses.fr/fr/system/files/ AP2008sa0408.pdf. Zugegriffen: 05. Aug. 2015

2. BAuA (2013) Photobiologische Sicherheit von Licht emittierenden Dioden (LED). L. Udovicic et al. http://www.baua.de/de/Publikationen/Fachbeitraege/F2115.pdf?_blob=publicationFile\&v=4. Zugegriffen: 05. Aug. 2015

3. Behar-Cohen F, Martinsons C, Viénot F, Zissis G, Barlier-Salsi A, Cesarini JP, Enouf O, Garcia M, Picaud S, Attia D (2011) Light-emitting diodes (LED) for domestic lighting: any risks for the eye? Prog Retin Eye Res 30(4):239-257

4. Blask DE (2009) Melatonin, sleep disturbance and cancer risk. Sleep Med Rev 13(4):257-264. doi:10.1016/j.smrv.2008.07.007

5. Cajochen C, Frey S, Anders D et al (2011) Evening exposure to alight-emitting diodes (LED)-backlit computer screen affects circadian physiology and cognitive performance. J Appl Physiol 110:14321438

6. DIN SPEC 67600 (o J) Fachbericht „Biologisch wirksame Beleuchtung - Planungsempfehlungen" (Ausgabe April 2013) Beuth-Verlag, Berlin

7. Figueiro MG, Wood B, Plitnick B, Rea MS (2013) The impact of watching television on evening melatonin levels, Article first published online: $1 \mathrm{JUL}$ 2013. doi:10.1002/j.2168-0159.2013.tb06297.x

8. Fritschi L, Erren TC, Glass DC, Girschik J, Thomson AK, Saunders C, Boyle T, El-Zaemey S, Rogers P, Peters S, Slevin T, D'Orsogna A, de Vocht F, Vermeulen R, Heyworth JS (2013) The association between different night shiftwork factors and breast cancer: a case-control study. Br J Cancer 109(9):24722480. doi:10.1038/bjc.2013.544

9. He C, Anand ST, Ebell MH, Vena JE, Robb SW (2015) Circadian disrupting exposures and breast cancer risk: a meta-analysis. Int Arch Occup Environ Health 88(5):533-547. doi:10.1007/s00420-0140986-x

10. SCENIHR (Scientific Committee on Emerging and Newly Identified Health Risks). Light Sensitivity. Opinion. http://ec.europa.eu/health/ph_risk/committees/04_scenihr/docs/scenihr_0_019.pdf. Zugegriffen: 23. Sept. 2008

11. SCENIHR (Scientific Committee on Emerging and Newly Identified Health Risks). Opinion: Health Effects of Artificial Light. http://ec.europa.eu/ health/scientific_committees/emerging/docs/ scenihr_o_035.pdf. Zugegriffen: 19. März 2012

12. Schierz C, Vandahl C (ohne Jahresangabe) Biologische Wirkung von Licht. TU Ilmenau, Fachgebiet Lichttechnik. http://www.m4ssl.npl.co.uk/wpcontent/uploads/2012/02/Protocol-on-Circadianeffective-radiation-DE.pdf. Zugegriffen: 05. Aug. 2015

13. Sigurdardottir LG, Valdimarsdottir UA, Mucci LA, Fall K, Rider JR, Schernhammer E, Czeisler CA, Launer L, Harris T, Stampfer MJ, Gudnason V, Lockley SW (2013) Sleep disruption among older men and risk of prostate cancer. Cancer Epidemiol Biomarkers Prev 22(5):872-879. doi:10.1158/1055-9965. EPI-12-1227-T

14. Sroykham W, Wongsawat $Y$ (2013) Effects of LEDbacklit computer screen and emotional selfregulation on human melatonin production. Conf Proc IEEE Eng Med Biol Soc 2013:1704-1707
15. Strahlenschutzkommission (2010) Moderne Lichtquellen, Stellungnahme der Strahlenschutzkommission. Verabschiedet in der 242. Sitzung der SSK am 01./02.Juli 2010. http://www.ssk.de/SharedDocs/Beratungsergebnisse/2010/Moderne_Lichtquellen.html. Zugegriffen: 05. Aug. 2015 